\title{
Effect of Aerobic Training and Resistance Training on Circulating Irisin Level and Their Association With Change of Body Composition in Overweight/Obese Adults: a Pilot Study
}

\author{
Hee-Jae KIM ${ }^{1}$, Hyo-Joo LEE ${ }^{1}$, Byunghun $\mathrm{SO}^{1}$, Jun Seok SON ${ }^{1}$, Donghyun YOON ${ }^{1}$, \\ Wook SONG ${ }^{1,2}$ \\ ${ }^{1}$ Health and Exercise Science Laboratory, Institute of Sport Science, Seoul National University, \\ Seoul, Korea, ${ }^{2}$ Institute on Aging, Seoul National University, Seoul, Korea
}

Received January 16, 2015

Accepted July 1, 2015

On-line October 8, 2015

\begin{abstract}
Summary
The novel myokine irisin has been reported as a therapeutic target for metabolic disease. The objective of this study is to reveal the effects of aerobic training (AT) and resistance training (RT) on circulating irisin levels and their associations with change of body composition in overweight/obese adults. Twenty eight overweight/obese adults (BMI $>23 \mathrm{~kg} / \mathrm{m}^{2}$ ) were included in this study and compared before and after 8 weeks of exercise program ( $60 \mathrm{~min} /$ day, 5 times in a week). The subjects, in both aerobic and resistance training, showed significant improvement in anthropometric parameters and exercise capacities including maximal oxygen uptake and muscle strength. Interestingly, the circulating irisin was significantly increased in resistance training group ( $p=0.002$ ) but not in aerobic training ( $p=0.426)$ compared to control group. In addition, we found the positive correlation between change of the circulating irisin and muscle mass $(r=0.432, p=0.022)$ and the negative correlation between change of the circulating irisin and fat mass $(r=-0.407, p=0.031)$. In the present pilot study, we found that circulating irisin level was increased by 8 weeks of resistance training in overweight/obese adults, suggesting that resistance training could be the efficient exercise type in overweight/obese considering positive change of body composition concomitant with increase of irisin levels.
\end{abstract}

\section{Key words}

Irisin • Myokin • Obesity • Aerobic training • Resistance training

\section{Corresponding author}

W. Song, Health and Exercise Science Laboratory, Institute of Sports Science, Seoul National University, 599 Gwank-ro,
Gwank-gu, Seoul 151-742, Korea. Fax: +82-2-872-2867. E-mail: songw3@snu.ac.kr

\section{Introduction}

Irisin, a novel myokine, is induced by the activation of PPAR $\gamma$ co-activator 1 alpha (PGC1- $\alpha$ ) which stimulates the fibronectin type III domain containing 5 (FNDC5) to cleave and release irisin into blood (Bostrom et al. 2012). In the previous report, the activation of irisin is triggered by exercise and it drives brown fat-like development of white adipose tissue (Bostrom et al. 2012). The thermogenic changes in white adipose tissue lead to the consideration that irisin would play a therapeutic role for obesity and type 2 diabetes. (Bostrom et al. 2012, Kelly 2012, Sanchis-Gomar et al. 2012, Villarroya 2012).

Since irisin was suggested as an exercise responsive myokine, several researches have been conducted whether FNDC5 and irisin are released at the onset of physical exercise (Bostrom et al. 2012, Hecksteden et al. 2013, Huh et al. 2012, Norheim et al. 2014, Pekkala et al. 2013, Timmons et al. 2012). Serum irisin and FNDC5 responded to acute and chronic exercises in murine (Aydin et al. 2014, Bostrom et al. 2012, Wrann et al. 2013). However, it was found that an inconsistency in the results as they all differently responded in humans according to the several studies (Bostrom et al. 2012, Hecksteden et al. 2013, Huh et al. 2012, Norheim et al. 2014, Pekkala et al. 2013, Timmons 
et al. 2012). Boström et al. (2012) reported an increased in circulating irisin level in healthy humans after 10 weeks endurance training, whereas other studies showed no significant changes of irisin level during chronic exercises. The studies reported that the serum level of irisin was not increased after 20 weeks of endurance training (Timmons et al. 2012), 12 weeks of endurance and strength combined training (Norheim et al. 2014), and 21 weeks of endurance training as well as after 21 weeks of endurance and resistance combined training (Pekkala et al. 2013).

Although the important role of exercise-induced irisin as a therapeutic target for obesity is well known, changes of circulating irisin levels following different types of exercise have not been fully explored. Moreover, considering the circulating irisin level responded by exercise training, research is needed on the effects of different types of exercise on circulating irisin level in obese population. Therefore, in the present pilot study, we investigated the effect of aerobic exercise and resistance exercise on circulating irisin level and their association with change of body composition in obese adults. These preliminary results serve to generate further hypothesis and facilitate the planning the exercise training program and management of participants.

\section{Methods}

\section{Recruitment and eligibility}

Between November and December 2012, 48 young overweight or obese adults (aged 19-35 years) were recruited from 113 students and staff email responders of the Seoul National University. The inclusion criteria required a $\mathrm{BMI} \geq 23 \mathrm{~kg} / \mathrm{m}^{2}$ or a body fat percentage $\geq 30 \%$ in women and $\geq 25 \%$ in men. Also, a stable body weight $( \pm 2 \mathrm{~kg})$ for 3 months before enrollment as well as a sedentary lifestyle (absence of moderate to vigorous regular physical activity in the past 3 months) were required. Subjects with diabetes, cardiovascular disease and asthma were excluded and as well as subjects with contraindications for exercise including decompensated heart failure, severe aortic stenosis, uncontrolled arrhythmia, and acute coronary syndromes.

Among 48 participants, 38 subjects were randomly assigned into aerobic and resistance exercise training group and 10 subjects were assigned to the control group. Sixteen subjects failed to complete the study after eight weeks of exercise intervention. The primary reason for drop out was the burden of time commitment. In addition, four sample data were excluded from the final analysis because the blood lipid characteristic exceeded normal ranges. The abnormal value, as our conjecture, could be resulted due to the different personal conditions which may have influenced the data. Two of them worked overnight prior to the visit and others had not been fasting according to the schedule. This study and the appropriate inform consents for study participants were approved by the Seoul National University Ethics Committee (SNUIRB 1210/001-003).

\section{Anthropometric and biochemical measurements}

Participants were evaluated before and after the exercise training. After an overnight fast, anthropometric measurements were evaluated for each study participant by the same examiner. The height was evaluated by using an extensometer and body weight, BMI, fat mass, body fat percentage, and skeletal muscle mass were measured by bio-impedance analysis using Inbody 370 (Biospace, Seoul, Korea) (Cho et al. 2013). Waist circumference was measured at the abdominal waist (smallest horizontal circumference above the umbilicus and below the xiphoid process), and hip circumference was measured at hips (horizontal, at maximal protrusion of gluteus maximus) as described in the American College of Sports Medicine (ACSM) Guidelines (Pollock et al. 2000). Subcutaneous fat thickness was measured by using the ultrasound device Body Metrix (Intela Metrix, CA, USA). Before taking a measurement, the device was put a small amount of auto sound gel on the front face. Gel on the device was spread out of 1 to 2 inch $(2.5-5 \mathrm{~cm})$ from the belly button. In addition, the device was moved up and down half inch $(1.25 \mathrm{~cm})$ with comfortable speed. The blood samples for lipid test and irisin were drawn after $12 \mathrm{~h}$ of overnight fasting and 2 days of minimal physical activity. The blood was drawn from the anticubital vein into precontained ethylenediaminetetraacetic acid (EDTA) tubes. Whole blood was centrifuged at $4{ }^{\circ} \mathrm{C}$ for $15 \mathrm{~min}$ at $3000 \mathrm{rpm}$ and then the separated plasma was stored at $-80{ }^{\circ} \mathrm{C}$ until subsequent analysis. The lipid profiles such as triglycerides (TG), total cholesterol (total-C), lowdensity lipoprotein cholesterol (LDL-C), high-density lipoprotein cholesterol (HDL-C), free fatty acid (FFA), glucose and insulin were measured at the Green Cross Reference Lab, Korea. The homeostasis model assessment-estimated insulin resistance (HOMA-IR) index was calculated as fasting insulin concentration $(\mu \mathrm{U} / \mathrm{ml}) \mathrm{x}$ fasting glucose concentration $(\mathrm{mg} / \mathrm{dl}) / 405$ 
(Kashiwabara et al. 2000). Plasma irisin (catalog\#EK067-16) was measured using a commercial Enzyme Linked Immune Sorbent Assays kit (ELISA, Phoenix Pharmaceuticals, CA, USA) and a micro-reader.

\section{Exercise protocol}

The exercise program was executed 5 days/week during 8 weeks at the dormitory gymnasium. The aerobic training program consisted of 60 -min sessions of whole body exercise. The initial duration of each exercise was gradually progressed to a maximum duration of $50 \mathrm{~min}$, based on the individual's capabilities. The training program was performed with $65-80 \%$ of the individual HRmax which was measured at baseline. The subjects alternated the two modes of aerobic exercise $(50 \mathrm{~min}$ treadmill only or the combination of $25 \mathrm{~min}$ cycling and 25 min treadmill mountain climber) each day during 8 weeks.

The resistance training program consisted of 60-min sessions of exercise for upper or lower body: six exercises for the upper body parts (shoulder press, seated rows, lat pull down, bench press, push up, pec deck fly) and five exercises for the lower body parts (squat, leg extension, leg curls, leg press, crunch). The upper body resistance exercise was performed on Monday and Wednesday; the lower body resistance exercise was performed on Tuesday and Thursday; and the upper and lower combined resistance training (bench press, lat pull down, leg curl, and leg press) was performed on Friday. The resistance load was $65-80 \%$ of one repetition maximum (1RM) testing which was performed at baseline and at the end of the study. The subjects were asked to perform 10-12 repetitions for 3 sets for all upper body and lower body exercises. Each exercise was performed in the moderate contraction velocity (2-3 s concentric, 2-3 s eccentric). The resting interval between sets was less than $1 \mathrm{~min}$. Weight was adjusted accordingly throughout the training program as the subjects' strength level gradually increased.

\section{Measurement of exercise capacities}

The isokinetic dynamometer (Humac Norm, CSMi, MA, USA) was used to measure the strength of the lower limbs. The isokinetic contraction test was taken to evaluate the knee extension and flexion torques of each lower limb. Prior to testing, participants were asked to perform two familiar practices for the test. After $10 \mathrm{~s}$ of resting, they performed five consecutive cycles of concentric knee extension and flexion at a speed of $60 \%$ s.
The grip strength was evaluated by using digital hand grip dynamometer (my-5401, TAKEI, Niigata, Japan). Participants were asked to stand with their arms and wrists in a neutral position. They were trained to perform a maximal isometric contraction by pulling the hand grip dynamometer with maximum effort. The test was repeated in both arms and the average value of four tests was used for the analysis.

In order to determine maximal oxygen uptake $\left(\mathrm{VO}_{2} \mathrm{max}\right)$, Bruce protocol was used on a treadmill ergometer as described in a previous study (Tanaka et al. 2001). The load was gradually increased by $3 \%$ incline and $2 \mathrm{~km} / \mathrm{h}$ after every $3 \mathrm{~min}$ from the start. A Treadmill T 150 Cosmed and Quark series by Breath Pulmonary Gas Exchange was used for functional diagnostics.

\section{Dietary intake}

The participants were required to report a threeday-dietary record during pre-, mid- and post- training. The recording days were comprised of one weekend day and two weekdays for each three-day-dietary recall period. The result of food intake was presented as the amount of total kcal per day. Prior to entering the exercise training program, the nutrition guideline for balanced meal was provided.

\section{Data analysis}

The results were expressed as mean $\pm \mathrm{SD}$. Homogeneity of participants among the three groups at baseline was calculated by One-way Analysis of Variance (ANOVA). The treatment effects (time) $\mathrm{x}$ (group) were determined by a repeated measure ANOVA. The adjusted $p$ value of less than 0.05 was taken to indicate a significant difference. The differences between final to basal level of variables were indicated as delta. To evaluate the correlation among the delta values, Pearson's $r$ test was conducted. Furthermore, multivariate regression analysis was conducted to adjustment for potential confounders. All analyses were performed using SPSS version 18.0 software (SPSS Inc., Chicago, IL, USA).

\section{Results}

A total of 28 subjects with a mean age 25.8 years and a mean BMI of $26.6 \mathrm{~kg} / \mathrm{m}^{2}$ were recruited. For aerobic and resistance training group, the average attendance rate in the exercise sessions was $87.4 \pm 4.3 \%$. The characteristics of the study participants before and 
Table 1. Characteristics of the study participants at pre- and post-training.

\begin{tabular}{|c|c|c|c|c|c|c|c|}
\hline & \multicolumn{2}{|c|}{$\begin{array}{c}\begin{array}{c}\text { Control } \\
(\text { total }=8,\end{array} \\
\text { Male=4, Female=4) }\end{array}$} & \multicolumn{2}{|c|}{$\begin{array}{c}\text { Aerobic training } \\
(\text { total }=10, \\
\text { Male }=6, \text { Female }=4)\end{array}$} & \multicolumn{2}{|c|}{$\begin{array}{l}\text { Resistance training } \\
\quad(\text { total }=10, \\
\text { Male }=7, \text { Female }=3)\end{array}$} & \multirow{2}{*}{$\begin{array}{c}\text { ANOVA } \\
p\end{array}$} \\
\hline & Pre & Post & Pre & Post & Pre & Post & \\
\hline \multicolumn{8}{|c|}{ Anthropometric characteristics } \\
\hline Age (years) & \multicolumn{2}{|c|}{$25.8 \pm 5.5$} & \multicolumn{2}{|c|}{$25.7 \pm 4.1$} & \multicolumn{2}{|c|}{$26.4 \pm 2.9$} & 0.425 \\
\hline Height $(\mathrm{cm})$ & $167.2 \pm 5.4$ & $167.5 \pm 5.5$ & $168.3 \pm 7.7$ & $168.5 \pm 7.8$ & $172.1 \pm 5.6$ & $172.1 \pm 5.7$ & 0.142 \\
\hline Body weight $(\mathrm{kg})$ & $74.1 \pm 7.6$ & $74.1 \pm 7.7$ & $75.3 \pm 12.7$ & $73.1 \pm 13.4^{*}$ & $80.3 \pm 12.8$ & $77.7 \pm 13.4^{*}$ & 0.030 \\
\hline $\begin{array}{l}\text { Skeletal muscle mass } \\
(\mathrm{kg})\end{array}$ & $32.8 \pm 4.9$ & $28.2 \pm 4.8$ & $28.4 \pm 6.4$ & $28.4 \pm 6.5$ & $32.5 \pm 4.8$ & $32.9 \pm 5.1$ & 0.271 \\
\hline $\begin{array}{l}\text { Skeletal muscle mass } \\
(\mathrm{kg}) / \text { Body weight }(\mathrm{kg})\end{array}$ & $0.38 \pm 0.0$ & $0.37 \pm 0.0$ & $0.37 \pm 0.0$ & $0.38 \pm 0.0^{*}$ & $0.40 \pm 0.0$ & $0.42 \pm 0.0^{* *}$ & $<0.001$ \\
\hline Body fat mass $(\mathrm{kg})$ & $23.1 \pm 4.0$ & $23.8 \pm 3.4$ & $23.7 \pm 4.8$ & $22.2 \pm 4.8^{*}$ & $22.1 \pm 7.3$ & $19.5 \pm 7.3 * *$ & $<0.001$ \\
\hline $\begin{array}{l}\text { Body fat percentage } \\
(\%)\end{array}$ & $31.1 \pm 5.7$ & $32.1 \pm 5.2$ & $32.4 \pm 4.8$ & $30.6 \pm 4.8^{* *}$ & $28.0 \pm 4.4$ & $24.7 \pm 5.1 * *$ & $<0.001$ \\
\hline$B M I(\mathrm{~kg} / \mathrm{m})$ & $26.5 \pm 2.0$ & $26.4 \pm 2.1$ & $26.4 \pm 2.4$ & $25.3 \pm 2.5 * *$ & $27.0 \pm 3.4$ & $26.0 \pm 3.6^{*}$ & 0.035 \\
\hline Waist $(\mathrm{cm})$ & $89.7 \pm 4.4$ & $85.5 \pm 6.7$ & $90.0 \pm 7.6$ & $80.9 \pm 8.8$ & $92.8 \pm 8.8$ & $84.6 \pm 10.6$ & 0.069 \\
\hline Hip (cm) & $104.2 \pm 3.2$ & $104.1 \pm 4.2$ & $104.9 \pm 4.2$ & $101.5 \pm 5.9$ & $105.0 \pm 5.9$ & $102.4 \pm 6.4$ & 0.077 \\
\hline$W H R$ & $0.86 \pm 0.0$ & $0.82 \pm 0.0$ & $0.86 \pm 0.0$ & $0.79 \pm 0.0$ & $0.88 \pm 0.0$ & $0.82 \pm 0.0$ & 0.351 \\
\hline $\begin{array}{l}\text { Subcutaneous fat } \\
\text { thickness ( } \mathrm{mm})\end{array}$ & $31.9 \pm 7.9$ & $33.3 \pm 3.2$ & $31.3 \pm 9.1$ & $27.4 \pm 6.6$ & $28.1 \pm 8.7$ & $25.3 \pm 8.2$ & 0.343 \\
\hline $\begin{array}{l}\text { Food intake } \\
\text { (kcal/day) }\end{array}$ & \multicolumn{2}{|c|}{$1509.11 \pm 88.47$} & \multicolumn{2}{|c|}{$1533.92 \pm 426.12$} & \multicolumn{2}{|c|}{$1575.65 \pm 227.09$} & 0.635 \\
\hline \multicolumn{8}{|l|}{ Blood parameters } \\
\hline Total-C (mg/dl) & $204.7 \pm 37.6$ & $203.1 \pm 34.6$ & $187.4 \pm 27.6$ & $182.1 \pm 25.8$ & $188.1 \pm 23.0$ & $169.0 \pm 24.6$ & 0.300 \\
\hline$L D L-C(m g / d l)$ & $124.2 \pm 28.8$ & $121.7 \pm 35.0$ & $112.5 \pm 29.5$ & $105.5 \pm 23.2$ & $116.7 \pm 26.0$ & $101.3 \pm 26.7$ & 0.362 \\
\hline$H D L-C(m g / d l)$ & $61.5 \pm 16.6$ & $62.6 \pm 16.7$ & $59.9 \pm 12.8$ & $63.3 \pm 12.1$ & $55.4 \pm 8.5$ & $54.6 \pm 13.0$ & 0.612 \\
\hline Triglycerides ( $\mathrm{mg} / \mathrm{dl})$ & $106.2 \pm 61.7$ & $120.0 \pm 74.9$ & $77.2 \pm 44.9$ & $87.0 \pm 26.3$ & $88.2 \pm 28.3$ & $91.3 \pm 37.7$ & 0.909 \\
\hline$F F A(\mu E q / l)$ & $452.2 \pm 181.6$ & $466.1 \pm 88.7$ & $435.3 \pm 108.5$ & $525.8 \pm 306.3$ & $458.8 \pm 135.8$ & $426.1 \pm 125.4$ & 0.483 \\
\hline Glucose (mg/dl) & $84.5 \pm 9.1$ & $90.3 \pm 9.4$ & $86.1 \pm 6.3$ & $85.8 \pm 7.0$ & $90.9 \pm 7.6$ & $87.4 \pm 9.2$ & 0.117 \\
\hline Insulin $(\mu U / m l)$ & $7.5 \pm 3.8$ & $13.2 \pm 9.1$ & $6.9 \pm 3.3$ & $9.5 \pm 4.3$ & $9.4 \pm 7.6$ & $8.9 \pm 5.9$ & 0.066 \\
\hline$H O M A-I R$ & $1.5 \pm 0.7$ & $3.0 \pm 2.2$ & $1.5 \pm 0.8$ & $2.0 \pm 0.9$ & $2.0 \pm 1.4$ & $1.9 \pm 1.4$ & 0.052 \\
\hline
\end{tabular}

Changes of anthropometric and blood profiles of the subjects were measured before and after 8 weeks of aerobic and resistance exercise training. Each value represents the mean $\pm \mathrm{SE}$. Total-C, total cholesterol; LDL-C, low-density lipoprotein cholesterol; HDL-C, high-density lipoprotein cholesterol. $* p<0.05, * * p<0.01$ indicate a significant difference between pre- and post-training values within group (paired t-test). ANOVA indicates two way repeated ANOVA measures between group and time.

after the exercise training were presented in Table 1. There were no significant differences among the three groups at the baseline anthropometric variables. However, after 8 weeks of exercise training, significant differences were found in body composition including body weight, body fat mass, BMI and relative skeletal muscle mass (adjusted by body weight) following 8 weeks of training in both of the exercise groups
(Table 1). In addition, there were no significant changes in blood profiles including cholesterols, triglycerides, free fatty acid, and insulin among the three experimental groups following exercise training (Table 1). Exercise capacities including isokinetic leg strength, hand grip strength and cardiovascular fitness $\left(\mathrm{VO}_{2} \max \right)$ were measured at pre- and post-training. There were no statistical changes in exercise capacities among the three 
Table 2. Change of exercise capacities of the study participants.

\begin{tabular}{|c|c|c|c|c|c|c|c|}
\hline & \multicolumn{2}{|c|}{$\begin{array}{l}\text { Control } \\
(\mathrm{N}=8) \\
\end{array}$} & \multicolumn{2}{|c|}{$\begin{array}{l}\text { Aerobic training } \\
\qquad(\mathrm{N}=10)\end{array}$} & \multicolumn{2}{|c|}{$\begin{array}{l}\text { Resistance training } \\
\qquad(\mathrm{N}=\mathbf{1 0})\end{array}$} & \multirow{2}{*}{$\begin{array}{c}\text { ANOVA } \\
p\end{array}$} \\
\hline & Pre & Post & Pre & Post & Pre & Post & \\
\hline \multicolumn{8}{|c|}{ Peak torque $60 \% \mathrm{~s}(\mathrm{~N} \cdot \mathrm{m})$} \\
\hline Extensor $R$ & $156.5 \pm 55.9$ & $152.6 \pm 61.2$ & $179 \pm 60.2$ & $169.8 \pm 57.6$ & $180.8 \pm 32.6$ & $193.5 \pm 31.3$ & 0.073 \\
\hline Flexor $R$ & $81.6 \pm 37.7$ & $83.1 \pm 30.5$ & $74.6 \pm 28.5$ & $84.4 \pm 32.5$ & $101.4 \pm 23.6$ & $107.8 \pm 19.9$ & 0.439 \\
\hline Extensor $L$ & $135.3 \pm 49.3$ & $142.8 \pm 58.2$ & $157.0 \pm 55.1$ & $161.3 \pm 59.7$ & $170.8 \pm 33.4$ & $184.8 \pm 28.9$ & 0.692 \\
\hline Flexor $L$ & $77.6 \pm 32.1$ & $81.0 \pm 21.1$ & $69.2 \pm 27.9$ & $79.9 \pm 28.4$ & $99.0 \pm 23.9$ & $102.2 \pm 22.0$ & 0.504 \\
\hline \multicolumn{8}{|c|}{ Peak torque $60^{\circ} / \mathrm{s} /$ Body weight $(\mathrm{N} \cdot \mathrm{m} / \mathrm{kg})$} \\
\hline Extensor $R$ & $207 \pm 55.7$ & $199.8 \pm 64.3$ & $232.5 \pm 44.7$ & $228.6 \pm 46.3$ & $226.9 \pm 38.7$ & $250.5 \pm 28.6^{* *}$ & 0.036 \\
\hline Flexor $R$ & $107.0 \pm 41.4$ & $109.3 \pm 30.5$ & $96.7 \pm 24.2$ & $112.7 \pm 25.5$ & $126.5 \pm 28.1$ & $138.8 \pm 18.2$ & 0.289 \\
\hline Extensor $L$ & $179.5 \pm 51.4$ & $186.5 \pm 59.4$ & $204.4 \pm 43.3$ & $215.1 \pm 48.1$ & $213.8 \pm 34.7$ & $239.6 \pm 29.4$ & 0.421 \\
\hline Flexor $L$ & $103.3 \pm 32.1$ & $106.5 \pm 32.1$ & $89.5 \pm 23.1$ & $106.8 \pm 20.4$ & $124.1 \pm 31.4$ & $133.7 \pm 24.2$ & 0.384 \\
\hline \multicolumn{8}{|c|}{ Hand grip strength (kg) / Body weight (kg) } \\
\hline & $28.9 \pm 9.1$ & $28.4 \pm 8.4$ & $35.2 \pm 9.9$ & $35.1 \pm 11.1$ & $37.1 \pm 7.0$ & $40.6 \pm 7.8^{* *}$ & 0.031 \\
\hline \multicolumn{8}{|c|}{$\mathrm{VO}_{2} \max (\mathrm{ml} / \mathrm{kg} / \mathrm{min})$} \\
\hline & $32.9 \pm 6.7$ & $30.7 \pm 6.9$ & $34.8 \pm 5.4$ & $37.9 \pm 4.9^{* *}$ & $36.1 \pm 2.9$ & $39.4 \pm 5.9 *$ & 0.023 \\
\hline
\end{tabular}

Exercise capacities including isokinetic leg strength, hand grip strength, and cardiovascular fitness were measured before and after 8 weeks of aerobic and resistance exercise training. $* p<0.05, * * p<0.01$ indicate a significant difference between pre- and posttraining values within group (paired t-test). ANOVA indicates two way repeated ANOVA measures between group and time.

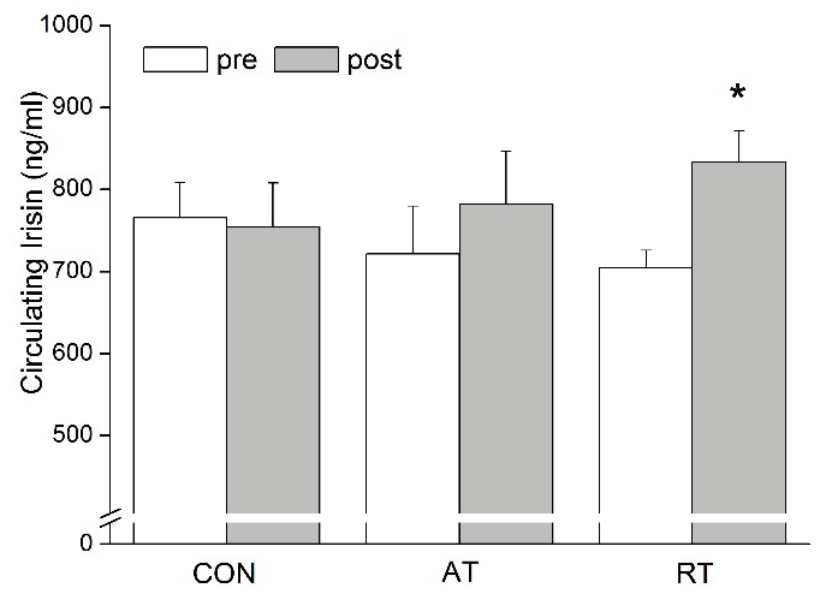

Fig. 1. Effect of 8 weeks of aerobic and resistance exercise training on circulating irisin level in control versus aerobic and resistance exercise training group. The white bar means pre exercise and black bar means post exercise training. * Indicate a significant difference between pre and post values $(p<0.05)$.

groups at the baseline. After 8 weeks of training, leg extensor muscle (right leg, adjusted with body weight) showed a significant improvement $(p<0.001)$ at $60 \%$ in resistance training group (Table 2). In addition, hand grip strength was significantly increased $(p<0.001)$ only in resistance training group (Table 2). The values of $\mathrm{VO}_{2} \max$ were improved in both of aerobic training $(p<0.001)$ and resistance training $(p<0.05)$ (Table 2).

The concentration of circulating irisin was measured at pre- and post-training. Compared to control group and aerobic training group, level of circulating irisin in resistance training group was significantly increased $(p<0.001)$ following 8 weeks of training (Fig. 1). As shown in Table 3, multivariate linear regression analysis adjusted with potential confounders including age, gender, body composition and food intake was performed. Significant correlations of irisin with exercise training persisted after adjustment for age, gender, skeletal muscle mass (baseline), fat mass (baseline), and daily calorie intake (Table 3). The alternation of circulating irisin level was correlated with increase in skeletal muscles $(\mathrm{R}=0.432, p=0.022)$ and decrease in fat mass $(\mathrm{R}=-0.407, p=0.031)$ (Fig. $2 \mathrm{~A}, \mathrm{~B})$. This result suggests that change of circulating irisin following exercise training was closely related to positive improvement of body composition in overweight/obese. 
Table 3. Multivariate regression analysis of anthropometric factors with change of circulating irisin levels as a dependent variable.

\begin{tabular}{|c|c|c|c|c|c|c|}
\hline & $\beta 1$ & $\beta 2$ & $\beta 3$ & $\beta 4$ & $\beta 5$ & $\beta 6$ \\
\hline Aerobic training & $0.048 *$ & $0.043^{*}$ & $0.046^{*}$ & $0.045^{*}$ & $0.037 *$ & $0.043^{*}$ \\
\hline Resistance training & $0.022 *$ & $0.023 *$ & $0.024 *$ & $0.021^{*}$ & $0.006^{*}$ & $0.007^{*}$ \\
\hline Age & & 0.196 & 0.171 & 0.230 & 0.056 & 0.059 \\
\hline Gender & & & 0.602 & 0.688 & 0.738 & 0.751 \\
\hline Muscle mass & & & & 0.280 & 0.109 & 0.113 \\
\hline Fat mass & & & & & $0.028 *$ & $0.030^{*}$ \\
\hline Food intake & & & & & & 0.754 \\
\hline
\end{tabular}

* Indicate significant correlation in multivariate linear regression adjusted with other variables $(p<0.05)$.

$\beta 1$ : unadjusted bivariate linear regression coefficient.

$\beta 2$ : multivariate linear regression coefficient adjusted for age.

B3: multivariate linear regression coefficient adjusted for age, gender.

$\beta 4$ : multivariate linear regression coefficient adjusted for age, gender, baseline skeletal muscle mass.

B5: multivariate linear regression coefficient adjusted for age, gender, baseline skeletal muscle mass, baseline fat mass.

$\beta 6$ : multivariate linear regression coefficient adjusted for age, gender, baseline skeletal muscle mass, baseline fat mass, food intake.

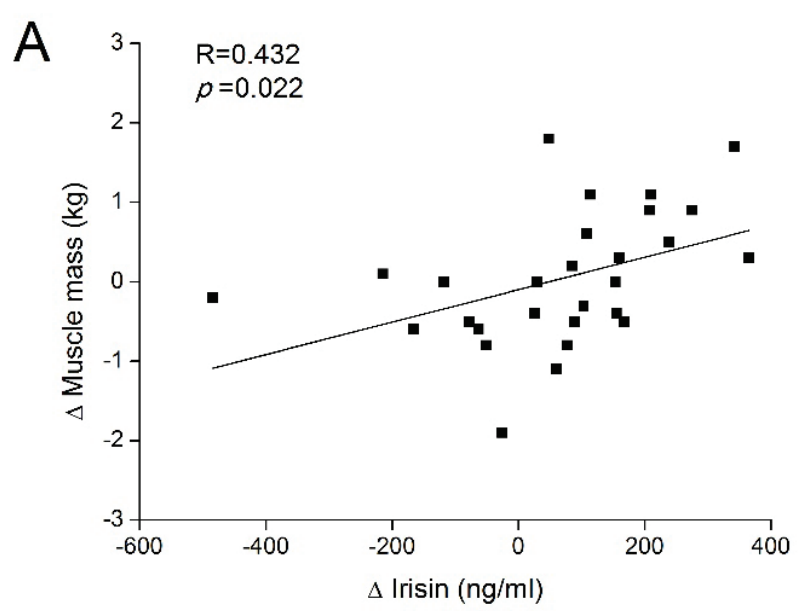

B

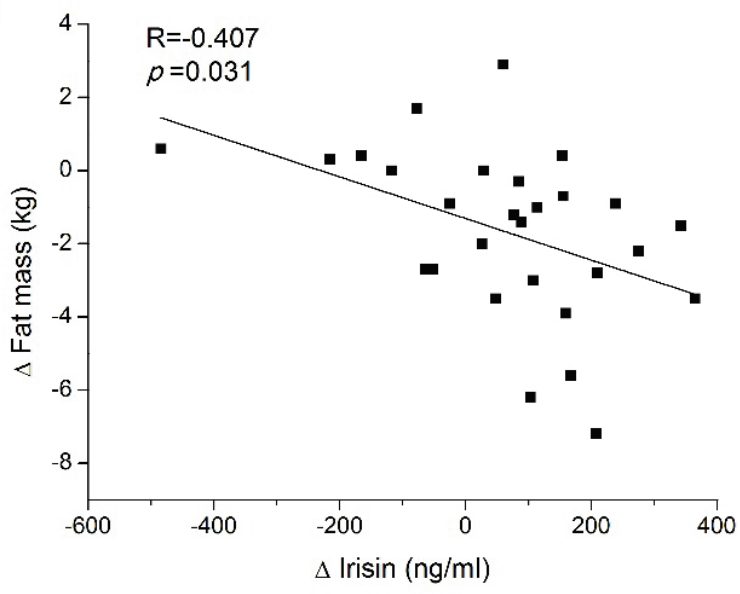

Fig. 2. Association of circulating irisin level with $(\mathbf{A})$ muscle mass $(\mathrm{r}=0.432, p=0.022)$, and $(\mathbf{B})$ body fat mass $(r=-0.407, p=0.031)$ in 28 overweight/obese adults after 8 weeks of exercise training.

\section{Discussion}

In the present study, circulating irisin protein concentration was significantly increased in 8 weeks of resistance training concomitant with positive improvement of body composition. And we found that alternation of circulating irisin was highly correlated with increase in skeletal muscle and decrease in fat mass. To our knowledge, this is the first study that investigated the effect of two different types of exercise training on circulating irisin and its association with body composition changes in overweight/obese adults.

Although irisin is known as exercise induced myokine, many studies carried out inconsistent results (Hecksteden et al. 2013, Huh et al. 2012, Norheim et al. 2014, Pekkala et al. 2013, Timmons et al. 2012). Timmons et al. (2012) reported a significant difference in FNDC5 mRNA only in the older subjects with endurance training from their experiment that analyzed gene chip data from 6 weeks of endurance training and 20 weeks of strength training in younger and older groups. From the result, Timmons et al. (2012) suggested that FNDC5 is not systematically affected by exercise. However, Boström et al. (2012) pointed out that the gene chip analyses showed smaller effect sizes and lack of increase

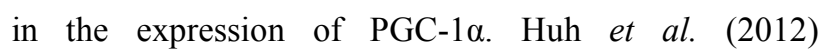
investigated acute and 8-week-lasting sprint training in healthy adults. Interestingly, the irisin levels were increased in acute training but remained unchanged in 8 weeks of training. Furthermore, similar to Huh et al. (2012), Norheim et al. (2014) concluded that 12 weeks of 
endurance training did not enhance the plasma concentration of irisin in an older group. They found that the expression of UCP1 mRNA is not correlated with the FNDC5 expression in subcutaneous adipose tissue or skeletal muscle. Hecksteden et al. (2013) also found an unchanged irisin level after 26 weeks of aerobic and strength endurance training. However, the interpretation of the study results was difficult. First of all, the training protocol (four or six bouts of $80 \mathrm{~m}$ sprints) in Huh et al. (2012) was an unusual preventive training that is different from the original work of Boström et al. (2012). Moreover, Norheim et al. (2014) found that increased FNDC5 mRNA by exercise was not translated into an increase in irisin circulation. Hecksteden et al. (2013) suggested that a degeneration of irisin level is possible in frozen samples. In this regard, our data with an unchanged circulating irisin level in aerobic exercise training showed a similar pattern to the previous studies. However, considering the key role of irisin as a myokine (cytokine which is produced, expressed and released by skeletal muscle), the result of a changed circulating irisin level in the resistance exercise training group seems meaningful. The result is similar to that in acute resistance training in obesity group (Pekkala et al. 2013). An interesting point is that the circulating irisin level was not changed by chronic strength training in healthy men (Hecksteden et al. 2013). Recently, Loffler et al. (2015) reported that increase irisin levels after acute strenuous exercise and 30-min bout of intensive exercise in children and young adults, whereas longer or chronic increases in physical activity did not affect irisin levels. There were differences compared to our experiments: 1) subjects (they recruited school children, whereas we recruited young adults), 2) intensity of exercise (they applied lowintensity physical activity, whereas we applied moderatevigorous intensity supervised training). Therefore, future studies are needed to investigate the effect of resistance exercise on circulating irisin in obese and healthy humans with the same study design.

To reveal an association between circulating irisin and obesity, several researchers investigated the correlation of circulating irisin with obesity parameters in humans. Huh et al. (2012) and Stengel et al. (2013) reported that circulating irisin showed a positive correlation with BMI. The researchers speculated that the positive association might be a compensatory mechanism. On the other hand, Choi et al. (2013) reported that circulating irisin showed a negative correlation with BMI. The differences among the studies might come from the diversity in subjects' characteristics and age. Although, several studies investigated the correlation of circulating irisin level with obesity, there is not enough research that shows different contribution by different type of exercise to the circulating irisin level in obesity. According to the recent studies by Boström et al. (2012) and Kelly (2012) irisin is likely to play a pivotal role in browning of white fat cell leading to heat production and energy expenditure. It also suggested that exercise induces muscle FNDC5 expression causing increased circulating irisin levels. Therefore, it is speculated that in our study, exercise training might have decreased fat mass and fat percentage which were potentially mediated by increased circulating irisin levels. In this study it could be noticed that circulating irisin level has a correlation with positive improvement of body composition in overweight/obese (Fig. 2). However, there is still lack of study to elucidate the complex association among exercise training, exercise-induced irisin levels, and change of body composition.

Limitations to the current study include: 1) small sample size and lack of consideration for gender difference, 2) high drop-out rate, 3) analysis of body composition by BIA, and 4) lack of evidence of browning marker. Firstly, our data have a lack of consideration for gender differences among the three groups. This limitation also includes the hormonal and metabolism influence in different gender. We additionally compared the genderdependent effect on irisin levels and there was no significant difference in men and women at baseline and their response to the training (data not shown). At the baseline, we designed the group by similar ratio of gender but the drop-out caused the gender differences at the final. Also, high drop-out rate was the limitation of the study. Total number of drop-out in each group was as follows; aerobic group (total $=7$, male $=5$, female $=2$ ) and resistance group (total $=8$, male $=2$, female $=6$ ). We compared the basic characteristics of participants and drop-out subjects in each training group. However, there was no significant difference between drop-out participants and training participants. Secondly, although several researches reported that BIA has a good agreement with DXA in measuring fat free mass, fat mass and body fat percentage (Fox et al. 1996, Pratley et al. 2000), BIA alone could not provide conclusive information of body composition that Dual Energy X-ray Absorptiometry (DEXA) could provide. Lastly, we were limited to estimate browning marker such as uncoupling protein 1 (UCP1), PR domain containing 16 (PRDM16) and other cytokines which might 
be related to circulating irisin level.

In conclusion, the findings of our research provided an insight for an approach to increase circulating irisin level in overweight/obese adults with resistance exercise training. In addition, although an association between exercise-induced irisin and body composition change was found in obese adults, we could not evaluate their causal relationship in this study. Therefore, the future study should cover the role of exercise-induced irisin on body composition including muscle mass and fat mass.

\section{Conflict of Interest}

There is no conflict of interest.

\section{Acknowledgements}

This work was supported by National Research Foundation of Korea (NRF) funded by Ministry of Science, ICT and Future Planning (NRF2013M3A9B6046417, NRF-2013M3A9D5072550, and MEST 2011-0030135) and Ministry of Education (NRF2014R1A1A2058645).

\section{References}

AYDIN S, KULOGLU T, AYDIN S, EREN MN, CELIK A, YILMAZ M, KALAYCI M, SAHIN I, GUNGOR O, GUREL A, OGETURK M, DABAK O: Cardiac, skeletal muscle and serum irisin responses to with or without water exercise in young and old male rats: cardiac muscle produces more irisin than skeletal muscle. Peptides 52: 68-73, 2014.

BOSTROM P, WU J, JEDRYCHOWSKI MP, KORDE A, YE L, LO JC, RASBACH KA, BOSTROM EA, CHOI JH, LONG JZ, KAJIMURA S, ZINGARETTI MC, VIND BF, TU H, CINTI S, HOJLUND K, GYGI SP, SPIEGELMAN BM: A PGC1-alpha-dependent myokine that drives brown-fat-like development of white fat and thermogenesis. Nature 481: 463-468, 2012.

CHO KO, JO YJ, SONG BK, OH JW, KIM YS: Colon transit time according to physical activity and characteristics in South Korean adults. World J Gastroenterol 19: 550-555, 2013.

CHOI YK, KIM MK, BAE KH, SEO HA, JEONG JY, LEE WK, KIM JG, LEE IK, PARK KG: Serum irisin levels in new-onset type 2 diabetes. Diabetes Res and Clin Pract 100: 96-101, 2013.

FOX AA, THOMPSON JL, BUTTERFIELD GE, GYLFADOTTIR U, MOYNIHAN S, SPILLER G: Effects of diet and exercise on common cardiovascular disease risk factors in moderately obese older women. Am J Clin Nutr 63: 225-233, 1996.

HECKSTEDEN A, WEGMANN M, STEFFEN A, KRAUSHAAR J, MORSCH A, RUPPENTHAL S, KAESTNER L, MEYER T: Irisin and exercise training in humans - results from a randomized controlled training trial. BMC Med 11: 235, 2013.

HUH JY, PANAGIOTOU G, MOUGIOS V, BRINKOETTER M, VAMVINI MT, SCHNEIDER BE, MANTZOROS CS: FNDC5 and irisin in humans: I. Predictors of circulating concentrations in serum and plasma and II. mRNA expression and circulating concentrations in response to weight loss and exercise. Metabolism 61: 1725-1738, 2012.

KASHIWABARA H, INABA M, MARUNO Y, MORITA T, AWATA T, NEGISHI K, IITAKA M, KATAYAMA S: Insulin levels during fasting and the glucose tolerance test and Homa's index predict subsequent development of hypertension. J Hypertens 18: 83-88, 2000.

KELLY DP: Medicine. Irisin, light my fire. Science 336: 42-43, 2012.

LÖFFLER D, MÜLLER U, SCHEUERMANN K, FRIEBE D, GESING J, BIELITZ J, ERBS S, LANDGRAF K, WAGNER IV, KIESS W, KÖRNER A: Serum irisin levels are regulated by acute strenuous exercise. $J$ Clin Endocrinol Metab 100: 1289-1299, 2015.

NORHEIM F, LANGLEITE TM, HJORTH M, HOLEN T, KIELLAND A, STADHEIM HK, GULSETH HL, BIRKELAND KI, JENSEN J, DREVON CA: The effects of acute and chronic exercise on PGC-1alpha, irisin and browning of subcutaneous adipose tissue in humans. FEBS J 281: 739-749, 2014.

PEKKALA S, WIKLUND PK, HULMI JJ, AHTIAINEN JP, HORTTANAINEN M, POLLANEN E, MAKELA KA, KAINULAINEN H, HAKKINEN K, NYMAN K, ALEN M, HERZIG KH, CHENG S: Are skeletal muscle FNDC5 gene expression and irisin release regulated by exercise and related to health? J Physiol 591: 53935400, 2013. 
POLLOCK ML, FRANKLIN BA, BALADY GJ, CHAITMAN BL, FLEG JL, FLETCHER B, LIMACHER M, PINA IL, STEIN RA, WILLIAMS M, BAZZARRE T: AHA Science Advisory. Resistance exercise in individuals with and without cardiovascular disease: benefits, rationale, safety, and prescription: An advisory from the Committee on Exercise, Rehabilitation, and Prevention, Council on Clinical Cardiology, American Heart Association; Position paper endorsed by the American College of Sports Medicine. Circulation 101: 828-833, 2000.

PRATLEY RE, HAGBERG JM, DENGEL DR, ROGUS EM, MULLER DC, GOLDBERG AP: Aerobic exercise training-induced reductions in abdominal fat and glucose-stimulated insulin responses in middle-aged and older men. J Am Geriatr Soc 48: 1055-1061, 2000.

SANCHIS-GOMAR F, LIPPI G, MAYERO S, PEREZ-QUILIS C, GARCIA-GIMENEZ JL: Irisin: a new potential hormonal target for the treatment of obesity and type 2 diabetes. J Diabetes 4: 196, 2012.

STENGEL A, HOFMANN T, GOEBEL-STENGEL M, ELBELT U, KOBELT P, KLAPP BF: Circulating levels of irisin in patients with anorexia nervosa and different stages of obesity - correlation with body mass index. Peptides 39: 125-130, 2013.

TANAKA H, MONAHAN KD, SEALS DR: Age-predicted maximal heart rate revisited. J Am Coll Cardiol 37: 153 156,2001

TIMMONS JA, BAAR K, DAVIDSEN PK, ATHERTON PJ: Is irisin a human exercise gene? Nature 488: E9-E10, 2012.

VILLARROYA F: Irisin, turning up the heat. Cell Metab 15: 277-278, 2012.

WRANN CD, WHITE JP, SALOGIANNNIS J, LAZNIK-BOGOSLAVSKI D, WU J, MA D, LIN JD, GREENBERG ME, SPIEGELMAN BM: Exercise induces hippocampal BDNF through a PGC-1alpha/FNDC5 pathway. Cell Metab 18: 649-659, 2013. 